\title{
AUTOLOGOUS TRANSFUSION
}

\author{
D Lee, J A F Napier
}

\section{Aims and techniques}

Aims of autologous transfusion:

- Reduce risks of transfusion

- Conserve stocks of blood

- Reduce work load in blood banks

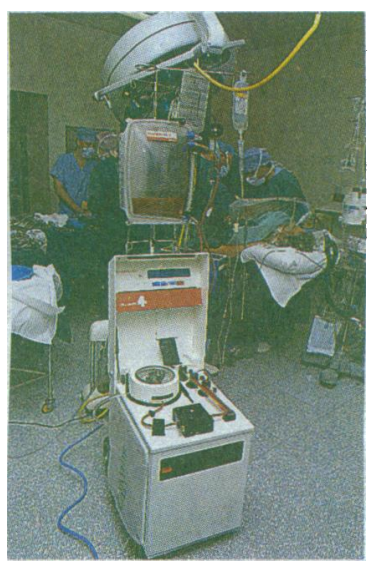

Cell saver in use during an operation.

\section{Risks of blood transfusion}

To reduce risks:

- Deposit autologous blood beforehand

- Use haemodilution before and during operation

- Use plasma expanders

- Salvage blood during operation
A much heralded alternative to the use of volunteer donations to replace blood lost during elective operations is the use of the patient's own blood. Autologous transfusion is primarily intended to avoid transmission of infection, in particular of HIV but also of the hepatitis viruses and other bloodborne pathogens. To be successful the autologous transfusion should ideally provide all the blood the patient needs. This benefit is reduced when the transfusion must be supplemented by the use of blood from volunteer donors. In seeking to reduce the risks it is important to consider other ways in which the aims might be achieved. These include a greater readiness on the part of clinicians to rely on synthetic volume expanders or albumin to replace blood losses and the use of planned haemodilution during operations. Most blood transfusions entail giving no more than two or three units of blood to replace comparatively small losses in patients who are not anaemic. This type of transfusion is potentially avoidable, and a reduction in the numbers would offer by far the greatest chance of reducing the overall risks of transfusion as well as relieving some of the pressure of work in blood banks, thereby increasing safety.

Autologous transfusion is a generic term encompassing various technical procedures. Two main approaches are possible; blood is either collected and stored during the weeks before operation or, alternatively, blood lost from the operation site is recovered and processed for transfusion - a procedure known as "blood salvage." In addition, though they are not strictly autologous there are also "directed" donations, which are collections of blood from family or friends to meet anticipated needs. To patients this approach is superficially attractive; experience has shown, however, that such donations are rather less safe than those collected from volunteer donors using the current stringent criteria for selection of donors. Friends and relatives are under such strong pressure to donate blood that they may not mention contraindicating factors. The practice of using directed donations is not endorsed by blood transfusion services, apart from the possible exception when a mother's blood may be used to transfuse her small infant.
Like all other therapeutic interventions, blood transfusion has risks as well as benefits. Until the advent of AIDS and the observed link between the transmission of HIV and transfusion, blood transfusion using blood collected from donors was regarded as a safe and reliable means of insurance in the event of massive operative blood loss. For a time after that, until the reliability of screening could be assessed, some people considered transfusion to be at best unsafe and at worst positively dangerous. 
The use of blood from volunteer donors is associated with risks, albeit small ones, and those risks associated with transmission of infection would not apply to autologous transfusions.

\begin{tabular}{|c|c|c|c|}
\hline \multirow[b]{2}{*}{ Risk } & \multicolumn{2}{|c|}{ Volunteer donors } & \multirow[b]{2}{*}{ Autologous blood } \\
\hline & Incidence & Mortality & \\
\hline Transmission of HIV & $1 / 10^{6}$ & $50 \%$ in 5 years & 0 \\
\hline Transmission of hepatitis B virus & $1 / 10^{5}$ & $5 \%$ & 0 \\
\hline \multicolumn{4}{|l|}{ Transmission of non-A, non-B } \\
\hline hepatitis virus & About $2 \%$ & Unknown & 0 \\
\hline Haemolytic transfusion reaction: & & $1 / 600000$ & \\
\hline $\begin{array}{l}\text { Resulting from clerical errors } 75 \% \\
\text { Resulting from technical errors } 25 \%\end{array}$ & & & $\begin{array}{c}\text { Still } \\
\text { possible }\end{array}$ \\
\hline Non-haemolytic transfusion reaction & $1 \%$ & $1 / 10^{5}$ & 0 \\
\hline Bacterial contamination & $2-5 / 10^{6}$ & About $100 \%$ & May be more likely \\
\hline
\end{tabular}

Clerical errors cause most of the deaths, however, and these are equally - if not more-likely to occur during autologous transfusion programmes. Some of the other sources of riskfor example, poor storage or bacterial contamination of units of blood - may also be more likely with autologous transfusion than with the use of donor blood supplied by the blood transfusion service. With our present limited experience of autologous transfusion these risks cannot yet be assessed accurately. Almost certainly the abdication by the blood transfusion service of responsibility for collecting and accurately labelling donations could lead to a risk of transfusion disasters from infected or mishandled units that could well exceed the present risk from donor blood.

\section{Responsibilities and organisation}

\author{
Clear instructions about \\ techniques and clerical procedures \\ must be laid down, and \\ responsibilities of all personnel \\ taking part must be defined.
}

There is evidence that people are showing greater readiness to take legal action when there is a possibility that a treatment may have had harmful consequences. In addition, the recent introduction of product liability legislation makes it even more important that approved procedures are clearly laid down and that evidence of compliance can be provided. It is therefore important for "predeposit" autologous transfusion programmes to have clear instructions laid down about the techniques and clerical procedures to be used, as well as definitions of the responsibilities of the personnel taking part. Guidelines have been prepared jointly by the British Blood Transfusion Service and the British Society for Haematology. It seems appropriate that the haematologists responsible for hospital transfusion policies should supervise autologous transfusion programmes when these are not organised through the blood transfusion services.

\section{Options for autologous transfusion}

Checklist for predeposit programme

- Determine the number of units of blood required

- Establish liaison between haematologists, blood bank, surgeons and anaesthetists, and the regional transfusion centre as necessary

- Plan to collect the blood during the five week period before the operation, the last collection being at least four days before, with collection intervals of about a week

- Confirm that the haemoglobin concentration exceeds $120 \mathrm{~g} / \mathrm{l}$ for men and $110 \mathrm{~g} / \mathrm{l}$ for women. Autologous transfusion is not advised if concentration is less than $100 \mathrm{~g} / \mathrm{l}$

- Obtain the patient's informed consent to the procedure

- Prescribe oral iron supplements

- Ensure correct labelling of containers of collected blood; these should be signed by the patient before collection begins

- Test a sample of the patient's blood for $A B O$ and Rh D group, screen the serum for atypical antibodies in case additional donor blood is needed, and screen the sample for hepatitis $B$ surface antigen and HIV antibodies
There are various ways in which patients can be offered autologous transfusion.

\section{Predeposit programmes}

Predeposit programmes require collection of blood donations during the weeks before operation. Up to four units can be collected at weekly intervals and stored conventionally at $4^{\circ} \mathrm{C}$. Even larger amounts can be accumulated by a system in which older units are replaced at the same time as fresh units are collected. If facilities for frozen storage are available (rarely the case in blood banks in the United Kingdom) units of blood can be collected and stored months or even years before elective operations. Frozen storage of blood as an insurance policy for healthy people for whom operation is not currently contemplated is, however, pointless, and commercial ventures preying on public anxieties cannot be condoned.

\section{Preoperative haemodilution}

Intraoperative haemoglobin concentrations well below normal are safe and in many cases may even be desirable. In the 48 hours before operation patients who are fit and not anaemic can have about a quarter of their blood volume withdrawn and replaced by a volume expander, thus making an equivalent amount of their own blood available to replace operative losses. 


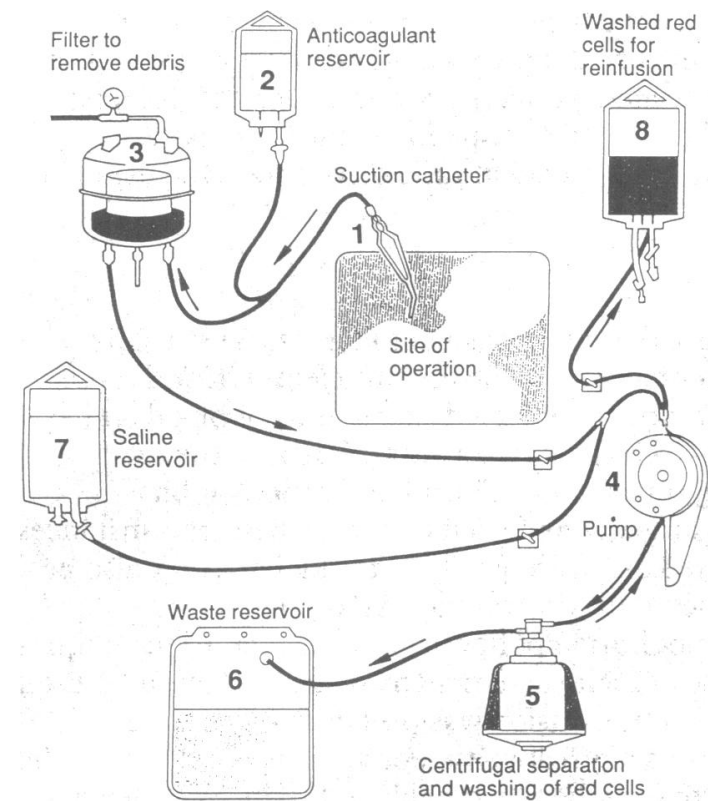

The component parts of the Haemonetics cell saver blood salvage system.

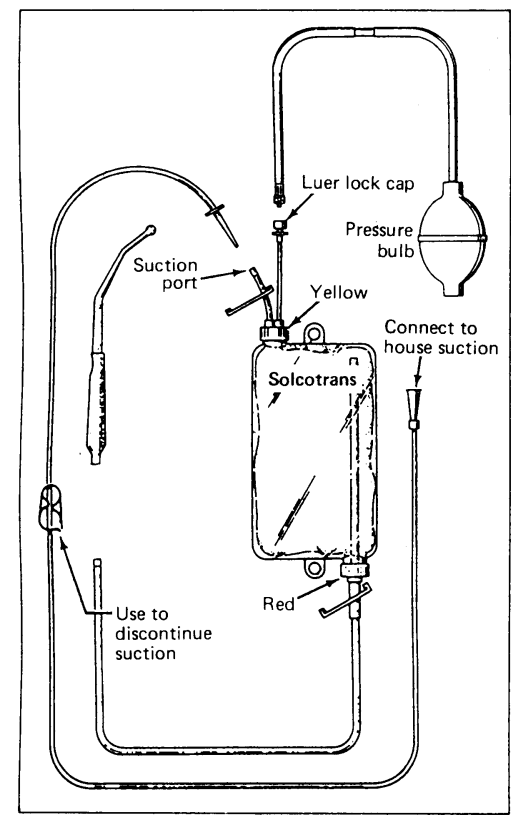

Solcotrans autologous whole blood transfusion system.
Blood salvage procedures

- Automated blood salvage - Several techniques are now available to recover shed blood for reinfusion. The operative site must be clean and free of bacteria, bowel contents, and tumour cells. The blood aspiration technique must avoid haemolysis from excessive suction, turbulence, and frothing. Popular in the United States, and being used more and more in the United Kingdom, is the Haemonetics "cell saver" equipment. Blood is aspirated and then anticoagulated and filtered to remove debris. The red cells are washed in a centrifugal cell washing system before being resuspended at a packed cell volume of about 0.50 for reinfusion. Two models now available are the Cell Saver 3 and the higher capacity Cell Saver 4, which is capable of processing about one unit of packed cells every three minutes. Either machine may be used in conjunction with homologous transfusion if large blood losses are expected. The Cell Saver 4 (which is highly automated in its collection and washing cycles) has been found to be invaluable in reducing the need for donor blood during major operations such as liver transplantation.

Although assembly of the disposable plastic software is quick, technical help must be available in the operating theatre. Recently a new portable model designed to cope with about one or two litres of blood has been introduced; this costs about $£ 6500$ (less than half the cost of the larger machines). With its lower running costs this equipment should be within the financial reach of an interested surgical unit. Plastic disposable items are comparatively expensive (about $£ 60$ a patient), particularly when the cost of collecting and processing donor blood is estimated at about $£ 20 /$ unit in the United Kingdom. Another disadvantage is that machines will sometimes be set up, and disposable items contaminated, when - because blood loss is less than expected - they are not required. Cell salvage machines are of greatest value when operative losses are unexpectedly high and cause a heavy drain on stocks in the blood bank.

- Manual systems for storage of red cells-An alternative approach is the Solcotrans autologous collection system. This comprises drainage/suction tubing linked by a $40 \mu \mathrm{m}$ blood filter to a specially designed reservoir containing acid citrate dextrose. Anticoagulated whole blood is collected and made reinfusable, and the system therefore differs from the washed red cells prepared by the Cell Saver system. Partial coagulation within the collection unit, or at least infusion of activated coagulation products, is a theoretical problem. Most published reports have been concerned with the transfusion of comparatively small numbers of units/patient, and disseminated intravascular coagulation and post-transfusion haemolysis of damaged red cells have not so far been problems. A variant of the system has been designed primarily for use in orthopaedics and entails collection and reinfusion of lost blood through drain sites after operation.

All blood salvage procedures result in containers of red cells being available for retransfusion. It is most important to ensure that collected units are labelled with patient's identification, the time and date of collection, and the maximum time allowable for reinfusion. It is not possible to avoid the ingress of micro-organisms, so the time between the start of collection and reinfusion should never exceed 12 hours.

\section{Predeposit autologous transfusion}

\section{Eligibility of patients}

Not every patient is suitable for autologous transfusion.

- The single absolute contraindication is active infection in the patient as coincidental bacteraemia could result in bacterial proliferation during storage.

- Anticipated needs-Only patients who have a reasonable expectation of needing transfusion should be considered for autologous transfusion. Any current local list of agreed maximum surgical blood orders may be used as a guide.

- Age and fitness-Fit patients between the ages of 18 and 65 who are free from cardiovascular, cerebrovascular, and respiratory diseases are the most suitable. Though autologous transfusion can be practised for patients outside these limits, the risks are greater and may well substantially exceed those from the use of donor blood. The fitness criteria used by the National Blood Transfusion Service should be used as guidelines. 
Children and adults weighing less than $45 \mathrm{~kg}$ will need proportionally smaller volumes of blood collected

\section{Conclusions}

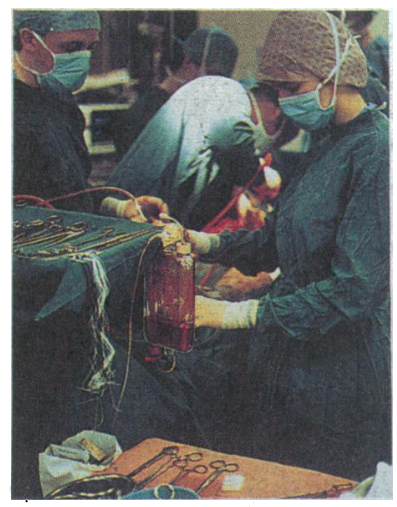

The whole blood transfusion system being used during a vascular operation (above) and in the ward after a total joint replacement (right).
- Pregnancy and autologous transfusion-Pregnant women can be autologous donors to cover expected delivery or surgical losses as long as they have no conditions associated with hypovolaemia (for example, preeclampsia). When the need for blood transfusion seems from past experience to be unlikely the risk to benefit ratio discourages the use of autologous transfusion.

It is important to recognise that predeposit autologous transfusion is an option available to few patients - those who are sufficiently fit and those who are expected to need comparatively modest amounts of blood. It has been estimated that these two categories constitute $5-10 \%$ of the total number of patients needing transfusion. Considerable motivation is required on the part of the surgeon, and even under the best circumstances autologous transfusion is used only rarely. The same patients may also be suitable for preoperative haemodilution or may avoid transfusion altogether if a planned haemodilution policy is in force. Manual techniques of blood salvage have limited capacity and in many instances have been used under circumstances in which transfusion was probably unnecessary. Automated blood salvage offers high capacity reprocessing. Both reduce the amount of crossmatching done by the blood bank and reduce the demand for donor units. In the most extensive blood replacement procedures, however, it is likely that some additional blood components or donor units may be required.

The decision to introduce an autologous transfusion programme requires the cooperation of haematologists, blood bank staff, the blood transfusion service, and surgeons and anaesthetists. A clear statement of the roles and responsibilities of each of the participants is necessary. Plans to introduce autologous transfusion in one form or another should be preceded by accurate costing and planned deployment of resources. Autologous transfusion should not be introduced without careful planning; under such circumstances a disaster would be particularly tragic because autologous transfusion is designed to reduce risk to the minimum.

The illustrations are published by courtesy of the following: the cell saver in use-Audiovisual Department, Withington Hospital, Manchester; the component parts of the cell saver system Haemonetics Ltd; and the diagram of the Solcotrans autologous whole blood transfusion system and the pictures of it in use-Solco Basle (UK) Ltd.

Dr D Lee is director, Regional Transfusion Centre, Manchester, and Dr J A F Napier is director, Welsh Regional Transfusion Centre, Cardiff.

\section{ANY QUESTIONS}

It seems to be agreed that the oestrogen containing combined pill should be stopped for at least four weeks before elective major surgery or surgery to the legs. ${ }^{1}$ Should the same policy invariably be followed for women taking oestrogens for climacteric symptoms, as queried in a recent letter?

Natural oestrogens affect haemostasis far less than the combined pill. Indeed, one comprehensive review boldly stated: "At present it may be concluded that oestrogen replacement therapy, per se, does not place the healthy postmenopausal woman at greater risk of developing arteriovenous thrombosis." 3 So far as I know there have been no subsequent reports of research to challenge this authoritative opinion. Moreover, if the oestrogen is given in a natural form and in a dose substantially equivalent to and replacing the function of functionless ovaries it could be argued that insisting on stopping such oestrogen would be comparable to requiring the castration of women in the late reproductive years as a prerequisite for major surgery.

The other side of the same argument, however, is that if a postmenopausal woman finds herself in a naturally safer state than she might be with either ovarian or replacement oestrogen she would do better to continue so. I would not exclude completely the possibility that a woman with some (known or unknown) predisposition to venous thrombosis may be made more vulnerable to this risk because of the added oestrogen. The stakes are lower than for the combined pill if hormone replacement is stopped for a few weeks: there is no fear of unwanted pregnancy, with its inevitable physical and emotional risks.

The risk of thrombosis increases with age, and some experts argue strongly that women over 45 years of age should normally receive some form of prophylactic antithrombotic cover for major surgery (Professor John Bonnar, personal communication, 1989). In these circumstances the hazards of continuing replacement treatment with natural oestrogens would surely be negligible in healthy women free of all other risk factors.

The fact that this treatment is in progress should be considered generally as a relative, not absolute, contraindication to proceeding with major surgery. A logical exception would be any woman who has recently (within one month) received an implant of $50-100 \cdot \mathrm{mg}$ oestradiol, as presently available implants produce a large surge in blood concentrations during the first weeks. The usual synergism with other risk factors such as obesity would apply - that is, more than one relative contraindication equates to an absolute contraindication. And in all cases in this age group you would also need a reason not to give heparin prophylaxis. - J GUILLEBAUD, medical director, Margaret Pyke Centre, London

I Guillebaud J. Surgery and the pill. BrMed F 1985;291:498-9.

2 Slater PA. Hormone replacement treatment. Br Med f 1989;299:51.

3 Notelowitz M, Ware $M$. Coagulation risks with postmenopausal oestrogen therapy. In: Studd JWW, ed. Progress in obstetrics and gynaecology. Edinburgh: Churchill Livingstone, 1982: $228-40$ 